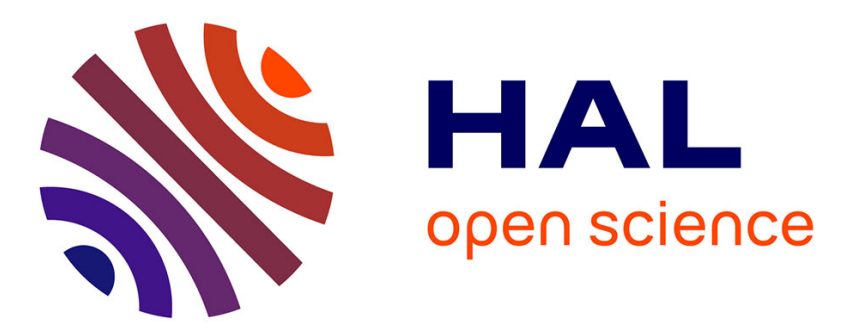

\title{
Modélisation analytique d'un système de pompage photovoltaïque utilisant une machine synchrone à commutation naturelle: fonctionnement optimal et critères de constitution
}

\author{
J.M. Rolland, Stéphan Astier, L. Protin, Ch. Masselot, M. Lajoie-Mazenc
}

\section{To cite this version:}

J.M. Rolland, Stéphan Astier, L. Protin, Ch. Masselot, M. Lajoie-Mazenc. Modélisation analytique d'un système de pompage photovoltaïque utilisant une machine synchrone à commutation naturelle: fonctionnement optimal et critères de constitution. Revue de Physique Appliquée, 1988, 23 (8), pp.1405-1414. 10.1051/rphysap:019880023080140500 . jpa-00245962

HAL Id: jpa-00245962

https://hal.science/jpa-00245962

Submitted on 1 Jan 1988

HAL is a multi-disciplinary open access archive for the deposit and dissemination of scientific research documents, whether they are published or not. The documents may come from teaching and research institutions in France or abroad, or from public or private research centers.
L'archive ouverte pluridisciplinaire HAL, est destinée au dépôt et à la diffusion de documents scientifiques de niveau recherche, publiés ou non, émanant des établissements d'enseignement et de recherche français ou étrangers, des laboratoires publics ou privés. 


\title{
Modélisation analytique d'un système de pompage photovoltaïque utilisant une machine synchrone à commutation naturelle : fonctionnement optimal et critères de constitution
}

\author{
J. M. Rolland ( $\left.{ }^{(}\right)$, S. Astier ( $\left.{ }^{1}\right)$, L. Protin ( $\left.{ }^{1}\right)$, Ch. Masselot ( $\left.{ }^{2}\right)$ et M. Lajoie-Mazenc $\left({ }^{3}\right)$ \\ ( $\left.{ }^{1}\right)$ Laboratoire d'Energie Solaire ENSUT (Ecole Nationale Supérieure Universitaire de Technologie), \\ B.P. 5085, Dakar, Sénégal \\ (2) Laboratoire d'Electrotechnique de Grenoble, ENSIEG (Ecole Nationale Supérieure d'Ingénieurs \\ Electriciens de Grenoble) à l'INPG, B.P. 46, 38 St Martin d'Hères, France \\ ( $\left.{ }^{3}\right)$ Laboratoire d'Electrotechnique et d'Electronique Industrielle, ENSEEIHT (Ecole Nationale Supérieure \\ d'Electrotechnique, Electronique, Informatique et Hydraulique de Toulouse) à l'INPT, France
}

(Reçu le $1^{\text {er }}$ avril 1987, révisé le 5 avril 1988, accepté le 9 mai 1988)

\begin{abstract}
Résumé. - Le système photovoltaïque étudié dans cet article est constitué par un moteur synchrone autopiloté entraînant une pompe centrifuge, associé à un onduleur de courant fonctionnant en commutation naturelle et alimenté au fil du soleil par un générateur photovoltaïque. Le fonctionnement optimal de ce système est défini et étudié analytiquement au moyen d'un modèle simplifié qui prend en compte tous les éléments de la chaîne de conversion. Dans le cadre des hypothèses retenues cette modélisation, effectuée en grandeurs réduites, permet de dégager et d'exprimer simplement la loi de commande et les critères électrotechniques à satisfaire pour la constitution d'un système de ce type. Ces critères portent sur les caractéristiques de la machine et son association au générateur. La démarche proposée, de portée générale pour l'étude des systèmes photovoltaïques, ouvre la voie pour une étude affinée du système considéré.
\end{abstract}

\begin{abstract}
The photovoltaic system presented in this paper is constituted of a self-piloted synchronous motor operating a centrifugal load and fed by solar cells through a load-commutated thyristors current inverter, without intermediate power storage. The optimal system working is defined and studied by means of a simplified analytical model which takes each item of the conversion chain into account, and is developped in reduced variables. Considering realistic hypotheses, this model allows to release the control law and to emphasize electrotechnical criterions to design and operate such a system. These criterions take effect on the machine constitution and its voltage matching with the generator. The proposed method is of larger utility for studying photovoltaic systems and gives way to a more accurate study.
\end{abstract}

\section{Introduction.}

L'exhaure de l'eau par énergie solaire photovoltaïque a constitué le thème de nombreux travaux ces dernières années [1]. Le choix d'un groupe immergé à courant continu à commutation électronique entraînant une pompe centrifuge répond bien aux contraintes associées à cette application, particulièrement pour les zones sahéliennes : sites hostiles et nappes profondes, maintenance difficile, adaptation d'impédance en temps réel nécessaire $[2,3]$.
L'utilisation dans ce type de groupe d'une machine synchrone à aimants magnétiquement rigides apporte, pour une robustesse équivalente à celle de la machine asynchrone, un meilleur rendement [4] ; de plus elle offre de larges possibilités d'adaptation à un fonctionnement particulier, par la variété des structures magnétiques réalisables [5].

Le dispositif étudié dans le présent article est constitué d'une machine synchrone autopilotée alimentée au fil du soleil (sans stockage d'énergie) par un générateur photovoltaïque au moyen d'un ondu- 
leur de courant à thyristors fonctionnant en commutation naturelle. L'intérêt a priori de ce dispositif pour l'application qui nous intéresse est sa bonne fiabilité résultant de la relative simplicité du convertisseur statique.

L'optimisation du transfert énergétique dans ce type de système dont la puissance de fonctionnement est essentiellement variable au cours de la journée doit répondre notamment à deux contraintes :

- adaptation optimale de l'impédance de charge du générateur pour assurer une bonne conversion photovoltaïque ;

- obtenir un bon rendement de la conversion électromécanique pour le fonctionnement en commutation naturelle retenu.

L'angle d'autopilotage de la machine constitue une variable d'entrée du système considéré, ce qui offre un degré de liberté pour l'optimisation du transfert énergétique. Avec ce seul degré de liberté l'optimisation du fonctionnement implique a priori un compromis entre les contraintes précédentes.

Nous nous proposons de montrer, dans l'étude qui suit, que le réglage de l'angle d'autopilotage peut permettre d'optimiser simultanément les deux conversions à condition qu'un certain nombre de critères électrotechniques de constitution du système soient réalisés.

Dans ce but nous utilisons un modèle analytique de l'ensemble de la chaîne de conversion du générateur à la charge. Ce modèle procède d'hypothèses simplificatrices mais réalistes eu égard aux critères recherchés, et permet de conduire une étude générale en grandeurs réduites du fonctionnement du système photovoltaïque.

\section{Le système et le dispositif expérimental.}

1.1 LE CONVERTISSEUR ÉLECTROMÉCANIQUE. Le système est représenté par le schéma de la figure 1. Il utilise une machine synchrone autopilotée associée à un onduleur de courant à thyristors fonctionnant en commutation naturelle. Rappelons que les signaux de commande $120^{\circ}$ du pont de thyristors sont alors synchronisés sur la vitesse de rotation du groupe. Ils déterminent les commutations du courant d'une phase à l'autre de la machine. La phase de ces signaux par rapport aux forces électromotrices à vide de la machine (fem) est réglable : c'est l'angle d'autopilotage ou calage affiché $\psi_{\mathrm{a}}$. Si l'on néglige la durée de la commutation, cet angle correspond au déphasage entre le fondamental du courant et la fem de la phase correspondante.

Dans les groupes classiques, ce convertisseur électromécanique (CEM) est usuellement alimenté à partir du réseau industriel par un générateur de courant continu $I_{\mathrm{c}}$ d'amplitude réglable, le calage étant alors réglé en fonction de $I_{\mathrm{c}}$ pour obtenir un fonctionnement au meilleur couple en assurant un angle de garde des thyristors minimal.

\subsection{ASSOCIATION AU GÉNÉRATEUR PHOTOVOLTAÏ-} QUE. - Dans notre application les courants $I_{\mathrm{c}}$ et les tensions $V_{\mathrm{c}}$ continus sont liés par la caractéristique électrique $I_{\mathrm{p}}\left(V_{\mathrm{p}}\right)$ du générateur photovoltaïque, qui n'est pas celle d'un générateur de courant, et qui dépend de l'énergie lumineuse incidente (éclairement) ainsi que le montre la figure 2 .

Pour un éclairement donné, ce générateur de

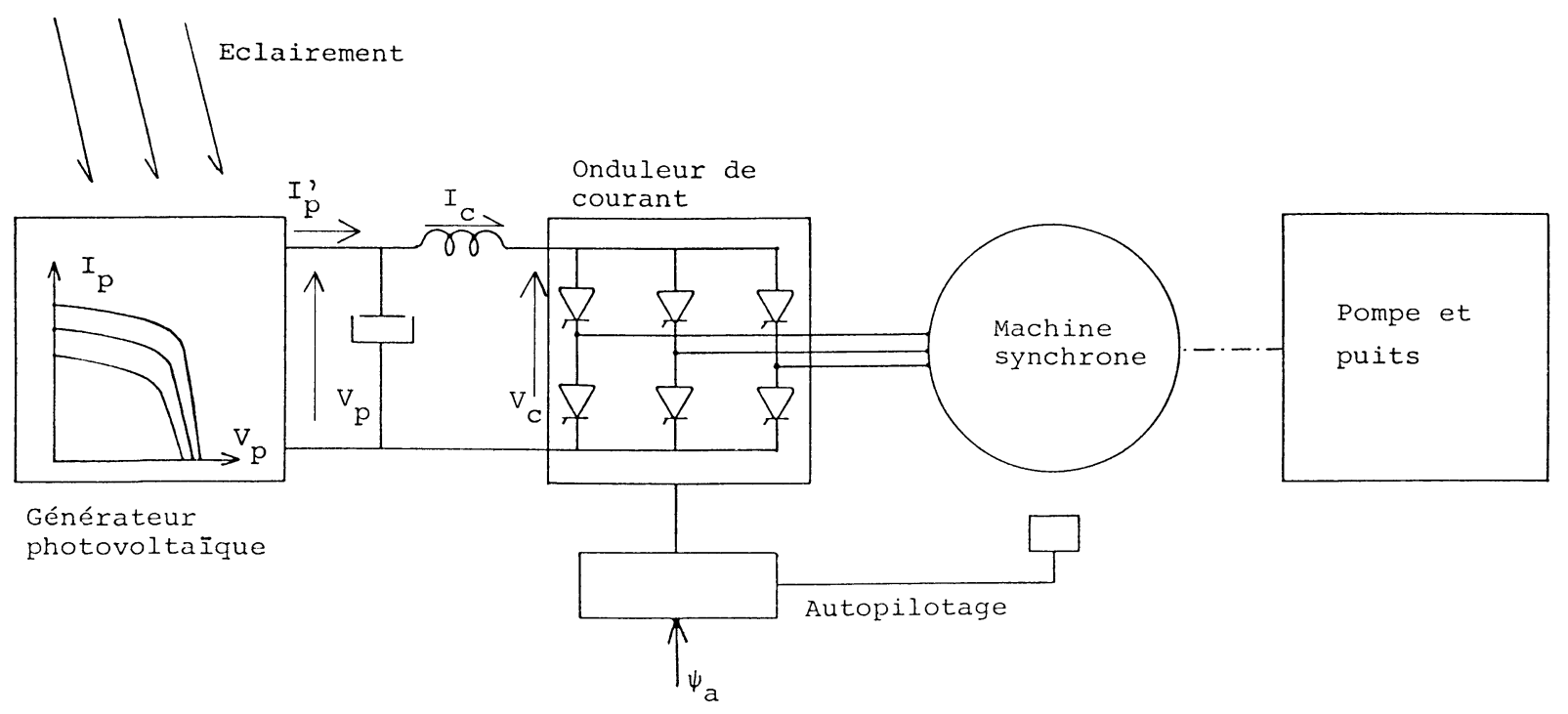

Fig. 1. - Schéma synoptique du système photovoltaïque.

[General diagram of the photovoltaic system.] 


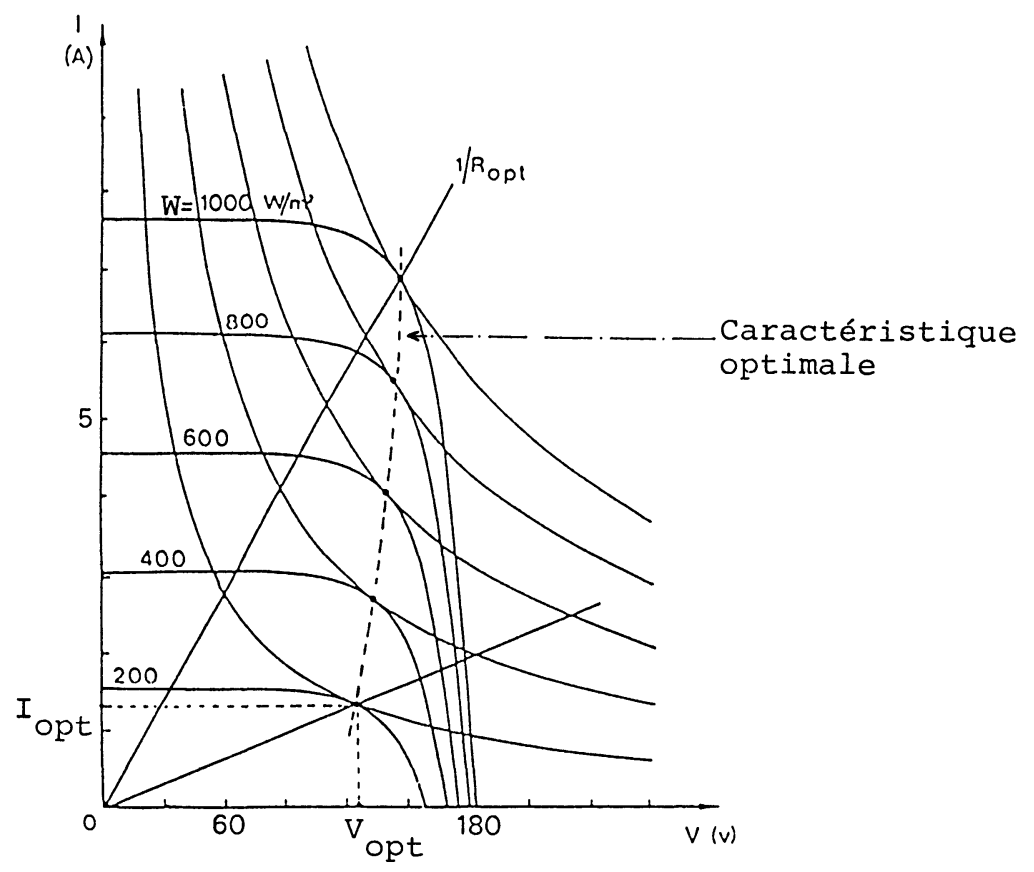

Fig. 2. - Caractéristiques électriques d'un générateur photovoltaïque haute tension pour différents éclairements $(W)$ et caractéristique optimale.

[A photovoltaic generator characteristics $I_{\mathrm{p}} v s . V_{\mathrm{p}}$ for various solar-ray powers $(W)$, and optimal characteristic.]

puissance finie fournit une puissance électrique maximale en un point de sa caractéristique qualifié d'optimal $\left(V_{\text {opt }}, I_{\text {opt }}\right)$. Lorsque l'éclairement varie, le lieu de ce point définit la caractéristique optimale du générateur, représentée sur la figure 2, [6]. L'association directe de l'ensemble convertisseur-machine au générateur photovoltaïque n'est pas admissible en raison de la non-réversibilité (instantanée) de ce dernier. Il est donc nécessaire de disposer une inductance $L$ en série avec le générateur qui absorbe les éventuelles excursions négatives de la tension redressée.

En outre les ondulations des grandeurs $v_{\mathrm{c}}$, $i_{\mathrm{c}}$ de l'étage continu se traduisent par une oscillation $\mathrm{du}$ point de fonctionnement sur la caractéristique du générateur qui entraîne une dégradation de la conversion photovoltaïque.

Le filtre $C, L$ inséré entre le générateur et l'onduleur permet de résoudre efficacement ce problème d'association, ainsi que l'illustrent les photographies 1 et 2 [7].

Ainsi dans la suite admettons-nous que les ondulations de tension et courant du générateur sont négligeables. La puissance instantanée $p=v_{\mathrm{p}} i_{\mathrm{p}}$ est alors égale à sa valeur moyenne $P=V_{\mathrm{p}} I_{\mathrm{p}}$.

1.3 LA CHARGE. - La charge du système est constituée par une pompe centrifuge débitant hors d'un puits de profondeur donnée. Dans le dispositif expérimental [8] celle-ci est simulée par une génératrice à courant continu qui, correctement réglée, permet d'approcher le comportement d'une pompe centrifuge fonctionnant en similitude [9]: couple résistant faible au démarrage et croissant rapidement avec la vitesse.

\section{Modèle et lois de commande.}

2.1 LE GÉNÉRATEUR PHOTOVOLTAÏQUE. - Les caractéristiques photovoltaïques sont représentées en figure 2 . Le courant de court-circuit $I_{\text {cc }}$ est proportionnel à l'éclairement alors que la tension à vide $V_{0}$ varie peu avec celui-ci. Dans la suite par abus nous appellerons éclairement la grandeur $I_{\mathrm{cc}}$, puisqu'elle en est une image.

On peut considérer que le courant optimal est luimême proportionnel à l'éclairement, ainsi :

$$
I_{\mathrm{opt}}=k_{\mathrm{i}} \cdot I_{\mathrm{cc}} \text {. }
$$

Nous admettrons également qu'en première approximation la caractéristique optimale du générateur est une droite à tension constante :

$$
V_{\mathrm{opt}}=k_{\mathrm{v}} \cdot V_{0} .
$$

2.2 LE CONVERTISSEUR ÉLECTROMÉCANIQUE. Pour la machine nous adoptons les hypothèses de Ben-Eschenburg (répartition sinusoïdale du champ dans l'entrefer, pôles lisses, linéarité magnétique).

Nous considérons de plus que sa résistance d'induit est négligeable et qu'elle est dotée d'amortisseurs 


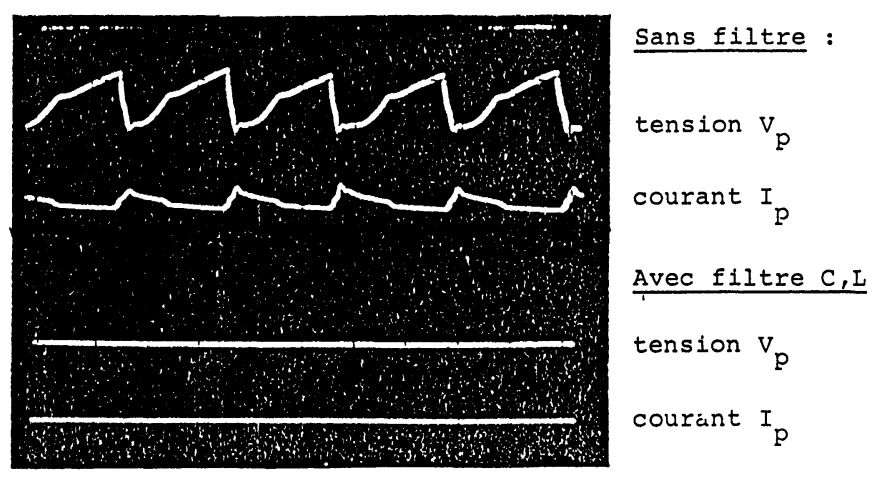

Photo. 1. - Ondulations des tension et courant du générateur expérimental : en association directe à l'ensemble commutateur-machine synchrone ; avec un filtre intermédiaire $C, L$.

[Experimental generator current and voltage : for direct coupling to the drive ; with a $C, L$ filter inserted.]

parfaits. Ce qui nous permet de négliger la durée de la commutation et permet de confondre $\psi_{\mathrm{a}}$ avec $\psi$, le déphasage entre la fem $E$ et le fondamental du courant $I$ dans la machine.

La contrainte de fonctionnement en commutation naturelle de l'onduleur est supposée saturée pour un angle de garde des thyristors $\alpha_{\mathrm{q}}=\omega t_{\text {inv }}=\omega t_{\mathrm{q}}$ constant $\left(t_{\mathrm{inv}}\right.$ étant le temps d'application de tension inverse aux bornes des thyristors et $t_{\mathrm{q}}$ leur temps de recouvrement).

Dans le cadre de ces hypothèses, le fonctionnement de la machine est déterminé par le diagramme de Ben-Eschenburg illustré en figure 3. Celui du convertisseur électromécanique peut alors être ana-

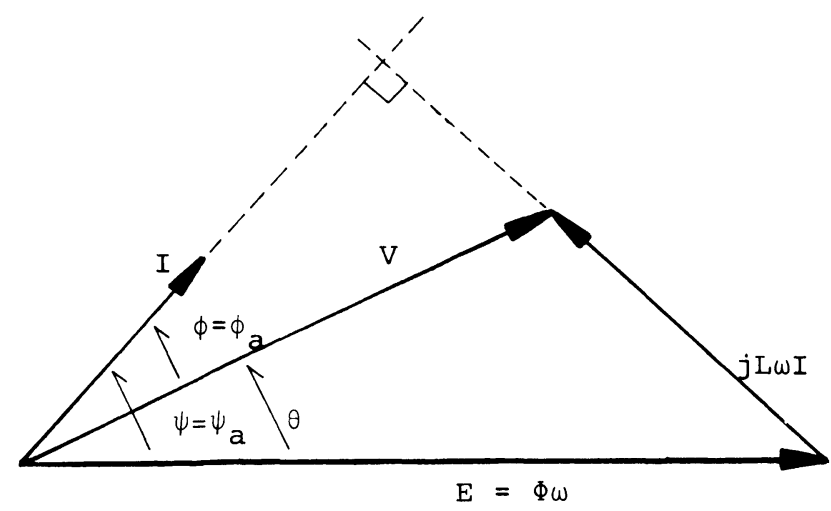

Fig. 3. - Diagramme de Ben-Eschenburg simplifié.

[Ben-Eschenburg diagram simplified.]

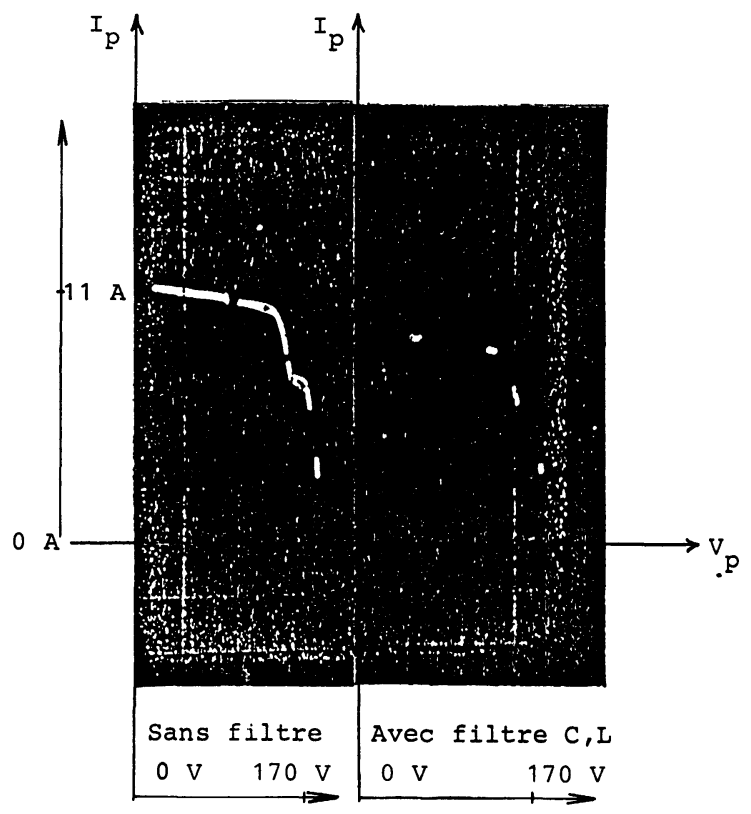

Photo. 2. - Oscillations du point de fonctionnement en différentes zones de la caractéristique du générateur photovoltaïque sans filtre et avec filtre $C, L$.

[Working points oscillations on various generator characteristic zones without filter and with a $C, L$ filter inserted.]

lysé selon le modèle d'un moteur à courant continu dont les balais sont décalables de $\psi_{\mathrm{a}}$ en avant de la ligne neutre [7] :

$$
\begin{gathered}
V_{\mathrm{c}}=\frac{3 \sqrt{6}}{\pi} \Phi p \Omega \cos \psi_{\mathrm{a}} \\
\Gamma=\frac{3 \sqrt{6}}{\pi} \Phi p I_{\mathrm{c}} \cos \psi_{\mathrm{a}}
\end{gathered}
$$

avec :

$I_{\mathrm{c}}=I_{\mathrm{p}}$ et $V_{\mathrm{c}}=V_{\mathrm{p}}$ les valeurs moyennes des courants et tensions côté continu de part et d'autre du filtre ;

$\Omega=\omega / p$ est la vitesse du groupe, avec $\omega$ la pulsation électrique d'alimentation et $p$ le nombre de paires de pôles de la machine ;

$\Gamma=$ est le couple électromagnétique moyen ;

$\Phi=$ est la valeur efficace du flux inducteur par phase de la machine et :

$E=\Phi \omega$ est la fem efficace par phase de la machine.

2.3 LA CHARGE. - Les caractéristiques mécaniques d'une pompe centrifuge font que sa vitesse varie avec la puissance $P_{\mathrm{a}}$ fournie à son arbre. Nous pouvons supposer qu'elle présente un fonctionnement dit en similitude à vitesse variable pour lequel sa caractéristique mécanique prend la forme usuelle :

$$
\Gamma=f \cdot \Omega^{2}
$$


où $f$ est une constante [7]

et

$$
P_{\mathrm{a}}=f \cdot \Omega^{3} \text {. }
$$

Dans ce cas le rendement manométrique de la pompe est constant lorsque la vitesse varie (invariant de Rateau) [9].

Le débit étant alors proportionnel à la vitesse on peut considérer que le maximum de débit est obtenu lorsque la puissance mécanique $P_{\mathrm{a}}$ fournie à l'arbre de la pompe est maximale.

2.4 OPTIMISATION ET LOIS DE COMMANDE. - Le système considéré ne dispose donc que d'un degré de liberté pour optimiser le transfert énergétique, par le biais du réglage du calage $\psi_{\mathrm{a}}$. L'optimisation consiste à régler $\psi_{\mathrm{a}}$ lorsque l'éclairement varie au cours de la journée pour obtenir un débit maximal à éclairement donné (ou une puissance électrique maximale délivrée par la génératrice de simulation). La loi de commande qui en résulte doit constituer le meilleur compromis entre les contraintes d'optimisation du fonctionnement propres à chaque élément de la chaîne de conversion : le générateur, le convertisseur électromécanique, la pompe centrifuge.

Compte tenu des propriétés de la pompe centrifuge, il s'agit donc de fournir une puissance maximale sur son arbre ce qui revient à optimiser le fonctionnement de l'ensemble générateur-convertisseur électromagnétique.

Loi optimale pour le générateur. - Des relations (1) à (5) nous extrayons la valeur du calage optimal $\psi_{\text {aopt }}$ à afficher pour que la charge du générateur impose à celui-ci un fonctionnement à puissance maximale fonction de son éclairement $I_{\mathrm{cc}}$ :

$$
\cos ^{3} \psi_{\mathrm{aopt}}=\left|\frac{\pi}{3 \sqrt{6}}\right|^{3} \frac{f}{\Phi^{3} p^{3}} \cdot \frac{k_{\mathrm{v}}^{2} \cdot V_{0}^{2}}{k_{\mathrm{i}} \cdot I_{\mathrm{cc}}} .
$$

Loi de fonctionnement au meilleur couple en commutation naturelle du CEM. - La contrainte de fonctionnement en commutation naturelle de l'onduleur s'exprime par:

soit

$$
\begin{gathered}
t_{\mathrm{inv}} \geqq t_{\mathrm{q}} \Rightarrow \Phi_{\mathrm{a}} \geqq \Phi_{\mathrm{amin}}=\omega t_{\mathrm{q}}=\alpha_{\mathrm{q}} \\
\psi_{\mathrm{a}} \geqq \psi_{\text {amin }}=\Phi_{\mathrm{amin}}+\theta .
\end{gathered}
$$

La valeur de $\psi_{\text {amin }}$ peut être déterminée par le diagramme de Ben-Eschenburg.

Afin de conduire une étude générale indépendante du bobinage de la machine introduisons les grandeurs réduites suivantes:

$i_{\text {red }}=I / I_{\mathrm{n}}$, valeur réduite du courant machine ramené à son courant nominal ; $I$ et $I_{\text {n }}$ sont les valeurs fondamentales efficaces de ces courants.

$L \%=L . I_{\mathrm{n}} / \Phi$ est l'inductance cyclique stator en $\%$ de la machine avec la fem à vide par phase de la machine comme tension de base [10] ; $L$ est l'inductance cyclique synchrone d'une phase de la machine.

Alors, en considérant que l'angle de garde $\alpha_{\mathrm{q}}$ des thyristors reste petit, ce qui permet de négliger les termes du second ordre, la contrainte de commutation naturelle s'exprime à partir du diagramme de Ben-Eschenburg par [7] :

$$
\begin{aligned}
\psi_{\mathrm{a}} \geqq & \psi_{\mathrm{amin}}= \\
& =\operatorname{Arcsin}\left[L \% i_{\text {red }}+\alpha_{\mathrm{q}} \sqrt{1-L \%^{2} \cdot i_{\text {red }}^{2}}\right] .
\end{aligned}
$$

La loi de commande $\psi_{\mathrm{a}}=\psi_{\text {amin }}$ correspond à un fonctionnement au meilleur couple en commutation naturelle.

Loi d'optimisation globale. - L'optimisation simultanée des fonctionnements du générateur et du CEM n'est a priori pas réalisable avec la seule grandeur de réglage $\psi_{\mathrm{a}}$. Comme nous négligeons les pertes Joule dans la machine, nous choisissons pour loi d'optimisation globale de l'ensemble générateurCEM :

$$
\psi_{\text {alc }}=\operatorname{Max}\left[\psi_{\text {aopt }}\left(I_{\mathrm{cc}}\right), \psi_{\mathrm{amin}}\left(i_{\text {red }}\right)\right] .
$$

Ce choix revient à privilégier le fonctionnement optimal du générateur tant que la commutation naturelle est assurée.

Nous nous proposons d'étudier le fonctionnement du système avec cette loi de commande à l'aide du modèle présenté et de dégager les critères de constitution et d'association qui en résultent.

\section{Harmonisation du modèle : caractéristiques rédui- tes.}

L'objectif étant de dégager des critères généraux portant notamment sur les caractéristiques de la machine, il est nécessaire de s'affranchir autant que possible du dimensionnement particulier à une installation, en introduisant des variables réduites adéquates.

L'expression (7) de la loi $\psi_{\text {amin }}$ ne dépendant pas du bobinage de la machine, elle satisfait cette condition. Il n'en est pas de même pour la relation (6) exprimant la loi optimale.

Nous avons donc été amenés à introduire un fonctionnement dit de référence permettant de dégager une écriture générale des équations de fonctionnement $\mathrm{du}$ système, notamment indépendant $\mathrm{du}$ couplage du générateur.

Considérons une installation donnée. Pour un couplage interne donné du générateur on peut choisir un point de fonctionnement optimal comme référence. Il lui correspond un éclairement de référence $I_{\text {ccref }}$ et une tension à vide $V_{\text {oref }}$.

Compte tenu de la nature du générateur et de la charge, à ce point unique de puissance maximale 
correspond une vitesse $\Omega_{\text {ref }}=\omega_{\text {ref }} / p$ du groupe telle que :

$$
V_{\text {cref }} \cdot I_{\text {cref }}=k_{\mathrm{i}} \cdot k_{\mathrm{v}} \cdot V_{\text {oref }} \cdot I_{\text {ccref }}=f \cdot \Omega_{\text {ref }}^{3},
$$

les pertes étant négligées.

Pour caractériser entièrement le système nous avons aussi été amenés à définir un coefficient d'adaptation générateur-machine permettant de caractériser l'influence du couplage du générateur relativement au bobinage de la machine :

$$
\beta=V_{\text {cref }} / E_{\text {cref }}
$$

où $E_{\text {cref }}=\frac{3 \sqrt{6}}{\pi} E_{\text {ref }}=\frac{3 \sqrt{6}}{\pi} \Phi \omega_{\text {ref }}$ correspond à la tension fictive constituée par la fem de la machine ramenée côté continu, c'est-à-dire la fem à vide redressée en onde entière $\left(\psi_{\mathrm{a}}=0\right)$.

Introduisons alors les grandeurs réduites suivantes pour le générateur :

$$
i_{\text {ccr }}=I_{\text {cc }} / I_{\text {ccref }} \text { et } v_{\text {or }}=V_{0} / V_{\text {oref }} .
$$

Ceci revient à réduire toutes les variables continues de fonctionnement par rapport aux grandeurs correspondantes de référence. Pour harmoniser toutes les variables électriques nous choisissons une liaison entre valeurs de référence côté machine et côté générateur.

Pour cela posons $I_{\text {ref }}=I_{\mathrm{n}}$ et conservons les règles de transformation :

$$
I_{\mathrm{ref}}=I_{\mathrm{n}}=\frac{\sqrt{6}}{\pi} I_{\mathrm{cref}}=\frac{\sqrt{6}}{\pi} k_{\mathrm{i}} I_{\mathrm{cc}} .
$$

Alors tous les courants réduits prennent dans chaque fonctionnement la même valeur $i$. Pour l'éclairement $I_{\text {ccref }}: i=1$, et le système absorbe sa puissance nominale. Des choix précédents résultent les valeurs de référence des tensions compte tenu de la relation (9), et en choisissant de surcroît :

$$
V_{\text {oref }}=\frac{\pi}{3 \sqrt{6}} \cdot V_{\text {cref }}
$$

toutes les tensions réduites ont même valeur $v$.

Nous imposons de plus $v_{\text {or }}=1$, ce qui supprime une information redondante au niveau couplage générateur/bobinage machine, car il suffit d'agir sur l'un ou sur l'autre pour choisir la valeur de $\beta$.

La loi de commande optimale qu'exprime la relation (6) s'écrit maintenant :

et

$$
\begin{aligned}
\cos ^{3} \psi_{\text {aopt }} & =\frac{\beta^{2}}{i}, \\
\cos \psi_{\text {aref }} & =\beta .
\end{aligned}
$$

De cette expression du calage optimal il résulte que $\beta<1$; ceci signifie concrètement que selon notre modèle à pôles lisses en commutation naturelle (fonctionnement démagnétisant de la machine) le couplage interne du générateur et son association avec le CEM sont nécessairement tels que le générateur soit basse-tension par rapport à la machine pour obtenir un fonctionnement optimal de celui-là.

La relation (7) s'exprime.:

$$
\sin \psi_{\mathrm{amin}}=L \% i+\alpha_{\mathrm{q}} \sqrt{1-L \%^{2} \cdot i^{2}}
$$

et la mise en œuvre de la loi globale $\psi_{\text {alc }}$ peut être effectuée sur la base d'une résolution graphique.

$\mathrm{La}$ figure 4 représente les lois de commande optimale et les contraintes de commutation naturelle pour différentes valeurs de $\beta$ avec $\alpha_{\mathrm{q}}=0$. L'exemple en trait gras montre la loi de commande globale d'un système pour lequel $\beta=0,5$ et $L \%=1$.

$\mathrm{La}$ caractéristique électrique du CEM avec contrainte de commutation naturelle saturée s'exprime à partir des relations (3) et (12). Moyennant une approximation du second ordre sur $\alpha_{\mathrm{q}}$, nous obtenons :

$$
\begin{aligned}
V_{\mathrm{c}}^{2}= & \left(\frac{3 \sqrt{6}}{\pi} \Phi \omega\right)^{2} \times \\
& \times\left(1-L \%^{2} \cdot i^{2} \cdot 2 \alpha_{\mathrm{q}} L \% i \sqrt{1-L \%^{2} i^{2}}\right) .
\end{aligned}
$$

Nous déduisons, tenant compte de l'équation (9) :

$$
\omega^{2} / \omega_{\text {ref }}^{2}=(v \cdot i)^{2 / 3} .
$$

Nous pouvons alors faire apparaître $\beta$ dans l'expression de cette caractéristique :

$$
\begin{aligned}
v_{\mathrm{ch}}(i)_{L \%, \beta}=\beta^{-3 / 2} \sqrt{i} & \left(1-L \%^{2} i^{2}-\right. \\
& \left.-2 L \% i \alpha_{\mathrm{q}} \sqrt{1-L \%^{2} \cdot i^{2}}\right)^{3 / 4} .
\end{aligned}
$$

Cette expression, que nous appellerons caractéristique électrique réduite du CEM, prend en compte les informations relatives à la totalité du groupe, charge mécanique comprise (cf. les relations (9) et (10)).

Une représentation graphique d'utilisation commode s'effectue à partir du changement de variable :

$$
i=i \quad \text { et } \quad v^{\prime}=\beta^{3 / 2} \cdot v .
$$

Alors les caractéristiques optimales du générateur s'écrivent :

$$
V_{\mathrm{opt}}^{\prime}=\beta^{3 / 2},
$$

et sont représentées (Fig. 5) par des droites verticales dont la position dans le plan électrique réduit est uniquement déterminée par le rapport d'adaptation $\beta$.

On peut aussi reporter dans ce plan les caractéristiques photovoltaïques. 


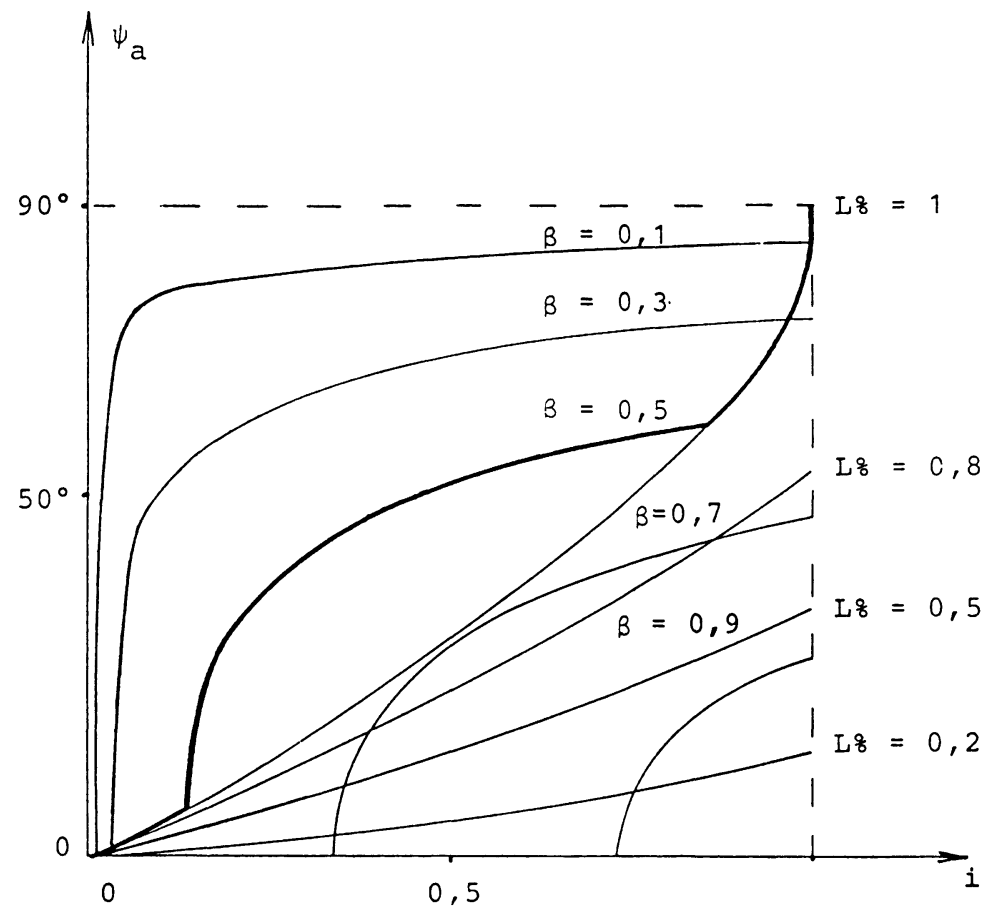

Fig. 4. - Lois de commande en grandeurs réduites : optimale, commutation naturelle $\left(\alpha_{\mathrm{q}}=0\right)$ et globale en trait gras.

[Representation in per cent values of optimal, natural commutation $\left(\alpha_{q}=0\right)$, and global control laws.]

D'autre part les caractéristiques du groupe s'expriment :

$$
\begin{aligned}
& v_{\mathrm{ch}}^{\prime}(i)_{L \%}= \\
& \quad=\sqrt{i}\left(1-L \%^{2} \cdot i^{2}-2 \alpha_{\mathrm{q}} L \% i \sqrt{1-L \%^{2} \cdot i^{2}}\right)^{3 / 4}
\end{aligned}
$$

et ne dépendent plus que de l'inductance $L \%$.

\section{Etude d'un système à l'aide des caractéristiques réduites.}

La figure 5 représente un réseau de caractéristiques de charge du système paramétrées en $L \%$ pour un angle de garde $\alpha_{\mathrm{q}}=10^{\circ}$.

Dans cette représentation les caractéristiques photovoltaïques optimales sont constituées par des droites à tension $V^{\prime}$ constante paramétrées en $\beta$.

4.1 ETUDE D'UN SYSTÈME EXISTANT; EXEMPLE EXPÉRIMENTAL. - L'évaluation des performances d'un système donné résulte de la comparaison des deux caractéristiques définies ci-dessus. Illustrons en trait gras le fonctionnement d'un système pour lequel $\beta=0,7$ et $L \%=0,2$ avec la loi globale définie précédemment.

Ce système n'est optimisable que sur un tiers environ d'une plage comprenant son courant nominal $i=1$.

- Pour $i=0,7$ la machine et le générateur fonctionnent simultanément de façon optimale.
- Pour $i>0,7$ l'adaptation d'impédance optimale générateur-machine impose un écart de calage que l'on pourrait évaluer sur un graphe du type de la figure 4. De cet écart découle pour la machine un déclassement supérieur à celui qu'impose la commutation naturelle.

- Pour $i<0,7$ la commutation naturelle empêche l'exploitation optimale de l'éclairement disponible. Physiquement, la vitesse du groupe est insuffisante pour que la tension redressée qu'il renvoie au générateur atteigne la valeur optimale $V_{\text {opt }}$, avec le réglage imposé par la commutation naturelle.

A titre expérimental nous avons étudié un montage quelconque constitué d'une machine didactique ordinaire alimentée par un générateur réel de $1,5 \mathrm{~kW}$ disponible en câblage haute-tension $(180 \mathrm{~V}$ à vide). Nous avons établi sur les bases du modèle, après identification des éléments du groupe, ses caractéristiques théoriques réduites dont les paramètres ont pour valeur $\beta=0,4$ et $L \%=0,6$. Celles-ci sont représentées en figure 6 dans les hypothèses $\alpha_{\mathrm{q}}=0^{\circ}$ et $\alpha_{\mathrm{q}}=10^{\circ}$.

Sur ce graphe apparaît tout d'abord la mauvaise adaptation entre le groupe d'étude et le générateur ; les valeurs $\beta=0,7\left(\alpha_{\mathrm{q}}=10^{\circ}\right)$ ou $\beta=0,8\left(\alpha_{\mathrm{q}}=\right.$ $\left.0^{\circ}\right)$ seraient adéquates : ceci nous a permis, considérant le bobinage de la machine correct, de redimensionner idéalement le générateur en puissance et tension.

Par ailleurs nous avons reporté sur la même figure 


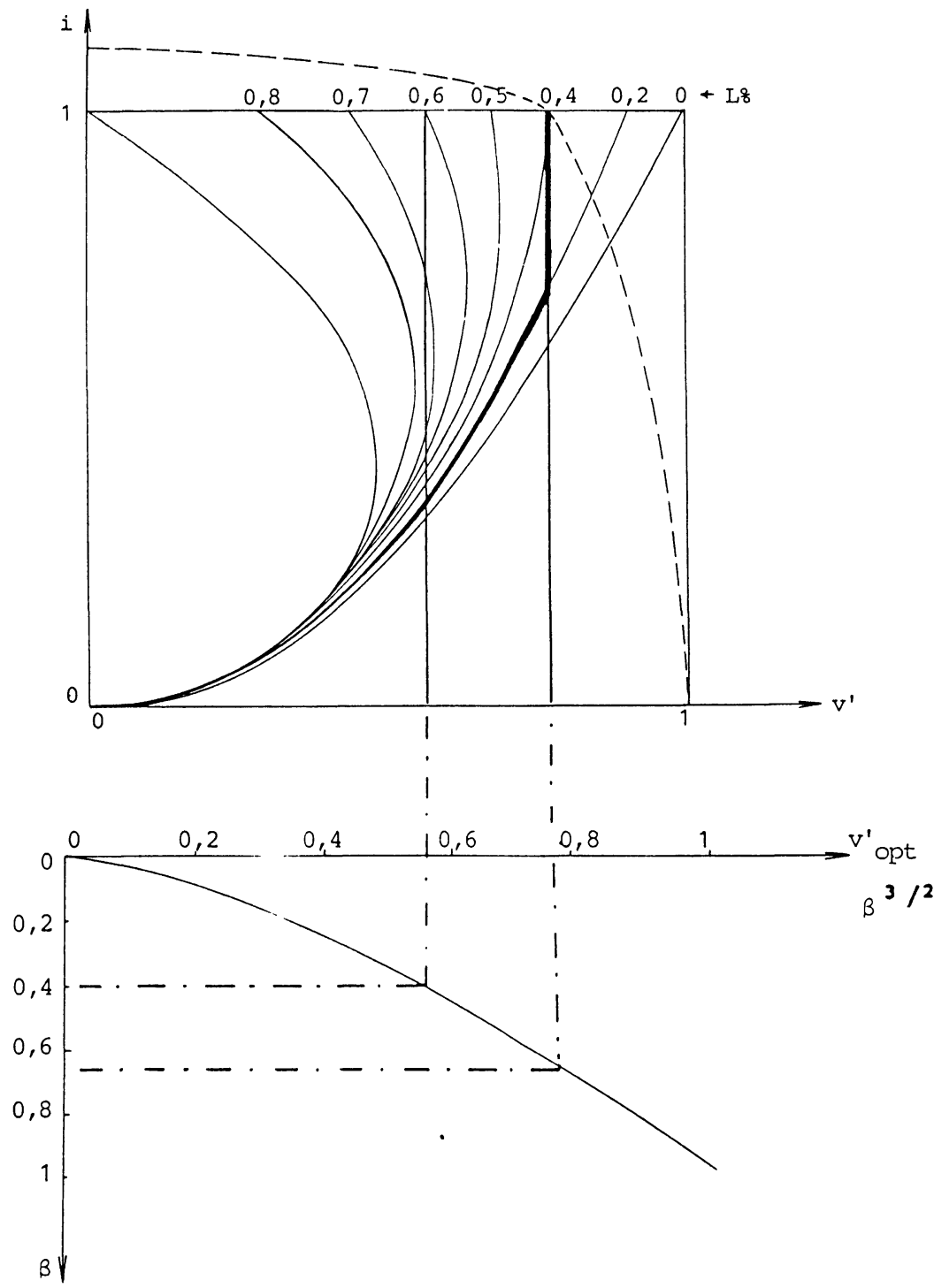

Fig. 5. - Caractéristiques électriques réduites du groupe en commutation naturelle avec $\alpha_{q}=10^{\circ}$ pour différentes valeurs d'inductance en pour cent $L \%$ de la machine. Caractéristiques optimales du générateur en fonction du couplage $\beta$.

[Representation in per cent values of natural-commutation-working characteristic $\left(\alpha_{\mathrm{q}}=10^{\circ}\right)$ of the drive for several $L \%$ per cent inductance values, and optimal generator characteristic as functions of the $\beta$ coupling ratio.]

les calages expérimentaux relevés, dont l'écart peu sensible avec les valeurs théoriques étaient prévisibles selon les approximations du modèle; cette courbe fait entre autres ressortir que l'éclairement plancher théoriquement exploitable $(i b=0,17)$ est en pratique $i b=0,18$. Enfin différents réglages sur le groupe ont permis de faire varier $\beta$ et $L \%$ et de vérifier les limites de fonctionnement du montage que le modèle prévoit.

4.2 Comparaison DE DifFÉRENTS Systèmes. Avec l'inductance $L \%=0,2$ de l'exemple précédent mais avec $\beta=0,4$ l'on voit sur la figure 5 que la plage d'optimisation possible est accrue; mais alors la machine est nettement plus pénalisée, pour l'utili- sation de son courant, que ne le nécessite la commutation naturelle, si l'on veut réaliser l'adaptation optimale au générateur. Pour le même rapport d'adaptation une machine d'inductance $L \%=0,6$ serait mieux adaptée. Par contre pour $L \%=0,8$ et $\beta=0,4$ le fonctionnement optimal n'est plus réalisable.

4.3 CONCEPTION D'UN SYSTÈME. - La meilleure adaptation relative des éléments d'un système est obtenue lorsque la caractéristique optimale du générateur coïncide au mieux avec celle de la charge. Ceci se présente pour :

- une machine d'inductance cyclique stator telle que $0,4<L \%<0,6$; 


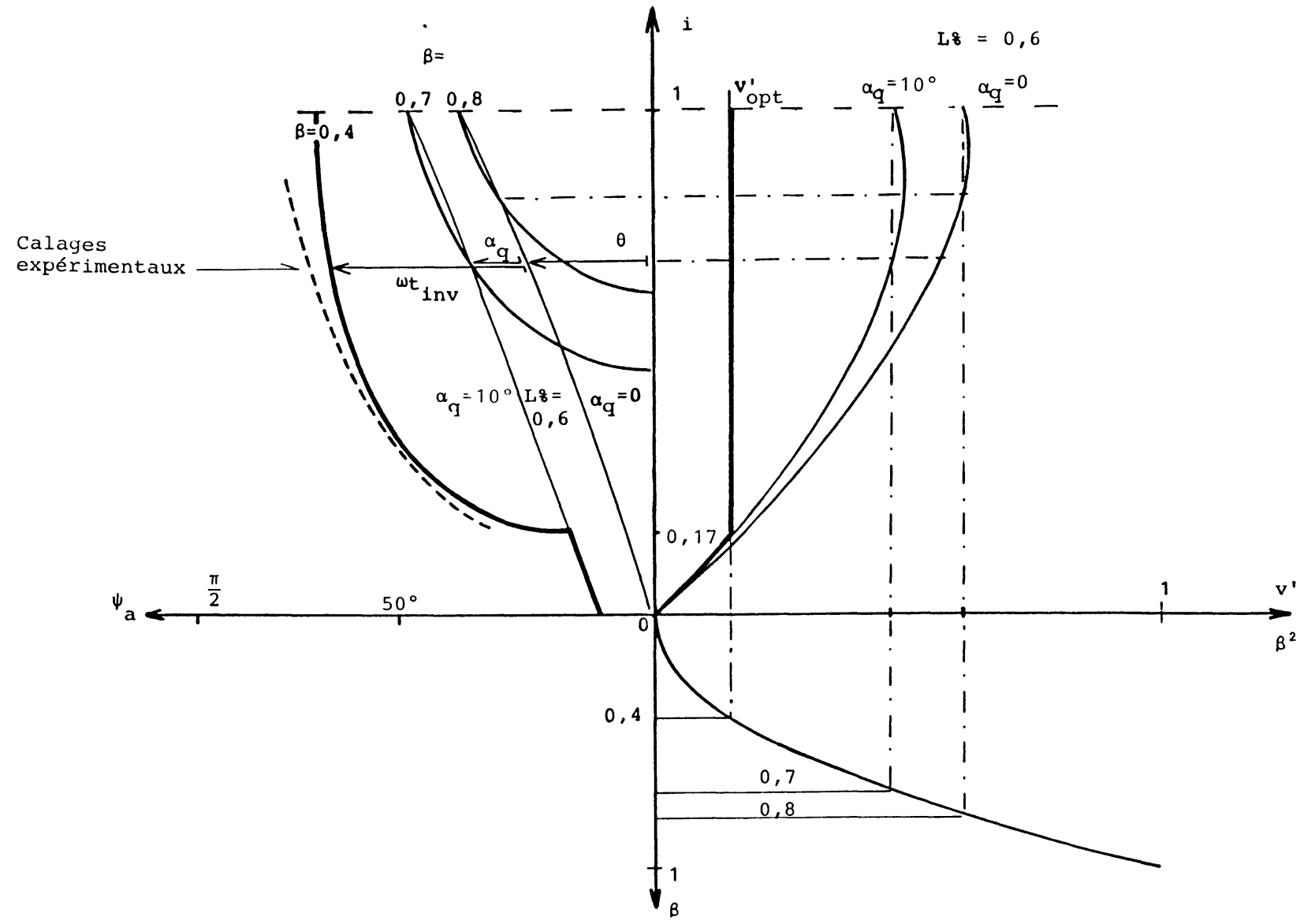

Fig. 6. - Caractéristiques théoriques réduites d'un groupe expérimental.

[Representation in per cent values of the theoretical characteristics of an experimental set-up.]

- une adaptation relativement haute tension de la machine par rapport au générateur de façon que $0,4<\beta<0,7$.

Dans ces conditions le déclassement de la machine imputable à l'adaptation de l'impédance de charge du générateur correspond sensiblement à celui qu'impose la commutation naturelle. Une plage d'optimisation plus ou moins large peut être obtenue avec de plus faibles valeurs de $\beta$ et $L \%$ au prix d'un plus grand écart de calage.

Les machines d'inductance $L \%>0,6$ ne s'adaptent optimalement au générateur qu'en aggravant leur déclassement déjà important compte tenu de leurs mauvaises performances en commutation naturelle.

Les machines d'inductance $L \%<0,4$ justifient l'adjonction au dispositif d'une deuxième grandeur de réglage assurant l'adaptation d'impédance. L'angle d'autopilotage peut alors être réglé pour bénéficier des bonnes performances de ce type de machine en commutation naturelle.

En définitive la conception d'un système de pompage tel que celui que nous considérons, doit prendre en compte les critères électrotechniques précédents qui portent essentiellement sur les caractéristiques du moteur synchrone utilisé et son adaptation au générateur. L'étude d'un système au cahier des charges déterminé peut être effectuée dans le cadre des hypothèses retenues sur la base des caractéristiques que nous avons introduites qui réunissent les informations sur l'ensemble de la chaîne de conversion énergétique :

- d'une part les caractéristiques $v_{c h}^{\prime}(i)_{L \%}$, relatives à :

- la construction géométrique, magnétique et thermique de la machine $(L \%)$;

- la contrainte de commutation naturelle $\left(\alpha_{\mathrm{q}}\right)$;

- la nature de la charge mécanique $(f, \operatorname{par} \beta)$;

- le couplage parallèle du générateur $\left(I_{\mathrm{cc}}\right.$ maximal) ;

- d'autre part les caractéristiques $v_{\text {opt }}^{\prime}$ qui rendent compte :

- du couplage série du générateur $\left(V_{0}\right)$;

- du bobinage statorique de la machine c'est-àdire de l'adaptation relative machine/générateur $(\beta)$. 


\section{Conclusion.}

L'utilisation d'un modèle analytique simplifié basé sur l'emploi de variables réduites convenablement choisies permet de conduire une étude générale du système photovoltaïque de pompage considéré qui met en œuvre un moteur synchrone à pôles lisses autopiloté entraînant une pompe centrifuge.

Il ressort essentiellement de cette étude que l'optimisation du fonctionnement du générateur photovoltaïque au cours de la journée entraîne des contraintes qui vont dans le même sens que celles imposées par le fonctionnement en commutation naturelle de l'onduleur. Ce type de machine paraît donc pouvoir être avantageusement exploité dans cette application de moyenne puissance, à condition de satisfaire les critères électrotechniques de conception dégagés par l'étude qui portent sur l'inductance et le bobinage.

L'inconvénient du réglage à un seul degré de liberté est en quelque sorte compensé par des conditions sur la conception de la machine et son couplage au générateur en fonction du cahier des charges : profondeur du puits, éclairement plancher à exploiter, dimensionnement de la pompe.

Ces conclusions valorisent le dispositif choisi pour sa relative simplicité et sa fiabilité et motivent une étude de faisabilité d'une machine à aimants permanents adaptée à ce dispositif. Une machine de structure $S P P$, sans pièces polaires, devrait pouvoir répondre aux spécifications imposées [10].

Soulignons d'autre part que bien que la méthode présentée néglige les pertes, celles-ci peuvent être prises en compte dans un deuxième temps, la méthode d'étude étant conservée. De même peut-on prendre en compte l'allure réelle des caractéristiques photovoltaïques optimales et la durée de la commutation par une exploitation numérique de la méthode proposée. Ces éléments ne modifient les résultats que sur le plan quantitatif, non qualitatif.

En revanche, cette étude ne permet aucune présupposition sur l'influence de la saillance polaire dont le rôle est souvent important dans les machines à aimants performantes [4].

\section{Bibliographie}

[1] Rapport de l'AFME (Agence Française pour la Maîtrise de l'Energie) Séminaire de contractants, Systèmes photovoltaïques (Sophia antipolis) 1985.

[2] Barlaud, M., Sow, G. et Masselot, Ch., Alimentation d'une pompe immergée à partir d'un générateur photovoltaïque à l'aide d'un onduleur triphasé à transistors, Revue Phys. Appl. 15 (1980) 263.

[3] MazurkiewiCZ, J., Submersible brushless motor with integrated electronics, Proc. PCI Motorcon (1983) pp. 542-548.

[4] Parallieu, L., Conception, réalisation et essais de machines synchrones à aimants utilisables dans des groupes motopompes immergés, Thèse de Docteur Ingénieur, INP Toulouse (1983).

[5] Lajoie-Mazenc, M., Leichter, S. et Astier, S., Machines à commutation électronique excitées par aimants magnétiquement rigides, $42^{\text {èmes }}$ Journ. parisiennes de la SEE (1980).

[6] Laugier, A. et Roger, J. A., Les photopiles solaires, Technique et documentation (1981).
[7] Rolland, J. M., Contribution à l'étude des groupes de pompage photovoltaïque, Expérimentation et analyse du fonctionnement d'un moteur autosynchrone à commutation naturelle alimenté par photopiles solaires, Thèse de Doctorat, INP Grenoble (1986).

[8] Rolland, J. M., Astier, S. et Masselot, Ch., A photovoltaic generator powering an underwater centrifugal pump coupled to a synchronous motor using a current inverter, Proc. ICEM 1986 - International Congress on Electrical Machines (1986) pp. 1145-1148.

[9] AGTHM (Association Générale des Hygiénistes et Techniciens Municipaux) Les stations de pompage d'eau, IEP Industries-Production/Environnement, Technique et Documentation (1981).

[10] AstiER, S., Contribution à la recherche des critères d'adaptation des machines excitées par aimants permanents à l'alimentation par convertisseurs statiques, Thèse de Docteur Ingénieur, INP Toulouse (1979) 